CONCURSO ESTUDIANTIL DE DISEÑO

\title{
Kuali: Distribuyendo comida por un bien
}

\author{
Julieta Navarro Rivera, Erika Yunuen Torres \\ Torres, Giovanny Alfonso Chávez Ceniceros, \\ Raymundo Cornejo
}

Publicado: 31 Octubre 2018

\begin{abstract}
Resumen
En este artículo se presenta un estudio preliminar sobre los retos y problemas que enfrentan los albergues, comedores, y diversas instituciones que proveen alimentos a distintas poblaciones en situación de vulnerabilidad. Mediante este estudio fue posible el diseño de una herramienta cuyo objetivo es facilitar la obtención y donación de alimentos y productos para evitar su posible desperdicio. De esta manera puede ser posible apoyar a instituciones que ofrecen alimentos y refugio a distintas poblaciones en situación de vulnerabilidad.
\end{abstract}

Palabras clave: Desperdicio de alimentos; poblaciones en situación de vulnerabilidad; aplicaciones móviles.

\section{Introducción}

En 2011, la Organización de las Naciones Unidas para la Alimentación y Agricultura (FAO por sus siglas en inglés, Food and Agriculture Organization) determinó que "aproximadamente un tercio de las partes comestibles de los alimentos producidos para el consumo humano se pierde o se desperdicia a nivel mundial, lo que equivale a alrededor de 1.300 millones de toneladas por año" [2]. La pérdida de comida involucra aquellos alimentos que no lograron llegar a la etapa de distribución en cualquier sub-etapa de la cadena de producción [1]. Por otro lado, el desperdicio de comida ocurre al final de la cadena de alimentos, en la venta y consumo de los mismos, donde el comportamiento del vendedor o consumidor conlleva a tirar o desaprovechar los alimentos [1].

Particularmente, Latinoamérica y el Caribe desperdician 15\% de los alimentos disponibles (127 millones de toneladas) [3,4]. De este $15 \%$, el $28 \%$ de los alimentos que se desperdician es por parte de los consumidores, mientras que otro $28 \%$ se desperdicia durante el manejo y almacenamiento del alimento por parte de los vendedores [3]. Es importante mencionar que de estos alimentos que se desperdician, aquellos que tienen los porcentajes más altos de desperdicio son frutas y vegetales (55\%), tubérculos (40\%), pescados y mariscos $(33 \%)$, y carne $(20 \%)$.

\footnotetext{
Navarro Rivera, J., Torres Torres, EY., Chávez Ceniceros, GA.,

Cornejo, R.

Universidad Autónoma de Chihuahua

Facultad de Ingeniería

Chihuahua, México

Email: a311012@uach.mx, a310886@uach.mx,a310831@uach.mx, rcornejo@uach.mx
}

Estos alimentos forman parte de los grupos básicos que proveen los nutrientes esenciales para la vida y desarrollo de las personas. Por lo tanto, el desperdiciar no solamente tiene implicaciones económicas en cuanto costos de producción o precios para el consumidor, también tiene un impacto social y humanitario. En consecuencia, la cantidad que se desperdicia de estos alimentos, y únicamente por los servicios que venden directamente a las personas, pudiesen ser alimentos aprovechados para alimentar alrededor de 36 millones de personas [4].

Por lo tanto, nuestro objetivo es ayudar a evitar este desperdicio de comida y facilitar su distribución a instituciones que proveen cuidados o servicios a poblaciones en situación de vulnerabilidad (albergues, casas hogar, comedores, centros de rehabilitación, etc.). Por lo tanto, el análisis de requerimientos se enfoca en el proceso que lleva al desperdicio durante la distribución (supermercados, mercados, etc.) y consumo (hogares, restaurantes) en la ciudad de Chihuahua, esto con apego a Ley para la donación altruista de alimentos del estado de Chihuahua [5].

Este trabajo se exploran los requerimientos de las instituciones de servicios y los donantes para comprender el proceso de adquisición de alimentos y se propone el diseño de una solución computacional que apoye este proceso.

\section{Trabajo relacionado}

Distintos trabajos se han enfocado en entender cómo distintas poblaciones en situación de vulnerabilidad utilizan la tecnología. Por ejemplo, Roberson y Nardi [6] explican cómo el personas sin hogar utilizan tecnología (i.e. teléfonos celulares, laptops, o cámaras) para registrar y comunicar espacios donde pueden encontrar refugio y alimento.

La ubicuidad y penetración de las tecnología de teléfonos inteligentes ha abierto la oportunidad para el desarrollo de aplicaciones que permitan explorar aún más los hallazgos de Roberson y Nardi. Por ejemplo, Mohan y Sarma [7] presentan un sistema de mensajería basado en textos para teléfonos celulares o inteligentes cuyo objetivo es incrementar el acceso a comidas calientes para aquellas personas sin hogar. Este sistema traslada el comportamiento fuera de línea de las personas al sistema en línea por el cual pueden compartir o recibir mensajes sobre aquellos lugares cerca de su ubicación y que están sirviendo gratuitamente comida caliente.

Los trabajos anteriores exploran los usos de la tecnología para fomentar la comunicación e inclusión entre las mismas personas sin hogar que necesitan alimento y refugio. Sin embargo, 
otros esfuerzos como GroceryMate [8] buscan generar empatía entre los consumidores de supermercados para incitarlos a donar alimentos nutritivos a quienes lo necesitan. El diseño de GroceryMate emplea tecnología persuasiva para tratar de generar empatía y se coloca físicamente en el carrito de compras. De esta manera, el consumidor puede observar el panel de aquellos productos que se recomiendan para su donación, así como la posibilidad de crear una lista de compras para simplificar el proceso de compras. Finalizada la compra, el consumidor cede los alimentos a la tienda para que ésta haga la donación a la organización que ayuda a las personas sin hogar. De manera similar al objetivo de GroceryMate, existen aplicaciones móviles que facilitan el acercamiento entre personas y organizaciones que necesitan algún Donativo. Bene Bene es una aplicación móvil que facilita la conexión entre personas que tienen algún objeto en buenas condiciones y que puede ser donado a alguna organización solidaria. El concepto básico es donar aquello que ya no le hace falta a la persona pero que que puede ser de beneficio a personas que lo necesitan y se encuentran cerca.

FoodForAll es otra aplicación móvil que permite a sus usuarios ordenar platillos de aquellos restaurantes o cafés cerca de ellos a precios accesibles. La reducción de estos precios promueve el consumo de aquellos alimentos que de otra manera se hubiesen desperdiciado. Aun cuando FoodforAll no precisamente promueve la donación de alimentos, esta aplicación ayuda a reducir el desperdicio de alimentos que se produce en el sector de restaurantes y cafés.

Estos esfuerzos toman ventaja del nivel de penetración tecnológica que hay en países desarrollados. Sin embargo, en países en desarrollo como México, muchas de las personas en situación de vulnerabilidad (e.g. personas sin hogar) carecen de acceso a estas conectividad en tiempo real. Sin embargo, es posible explorar soluciones tecnológicas que permitan conocer las necesidades de las organizaciones solidarias y motivar la donación de alimentos. Por lo tanto nuestro enfoque es explorar lo anterior en tres niveles: individuos, restaurantes, y corporativos y así lograr disminuir el desperdicio de comida pero aún más importante lograr un diseño que ayude a aquellas organizaciones que proveen de alimento nutritivo a personas en situación de vulnerabilidad.

\section{Metodología}

La investigación siguió un enfoque centrado en el usuario llevado a cabo con tres instituciones que proveen gratuitamente alimentos a personas en la ciudad de Chihuahua, México. Para este estudio se realizaron entrevistas con cada uno de los administradores de cada institución que se encargan de administrar y solicitar donativos de alimentos. Estas entrevistas fueron semiestructuradas con el objetivo de entender el proceso de solicitar y recibir donativos de alimentos, sus retos principales, y necesidades primarias. Durante las entrevistas se alentó a las personas a describir los procesos de donaciones, qué tipo de servicio proveen a la población y sus mayores necesidades como institución donataria.Adicionalmente, se les pidió a los participantes (responsables de las instituciones) a describir situaciones inusuales, artefactos que utilizan para solicitar y recibir donativos, y el tipo de poblaciones que atienden. Se obtuvo consentimiento verbal de cada uno de los participantes para grabar en audio la entrevista, así como el uso registrar en fotografías información adicional (véase Figura 1). La primer entrevista se llevó a cabo en el Albergue San Vicente de Paul A.C., el cual da alojamiento y alimentos a familias foráneas de escasos recursos. Estas familias solicitan la ayuda de este Albergue debido a que se trasladan a la ciudad de Chihuahua para recibir algún tipo de atención médica. La segunda entrevista se llevó a cabo en la Casa Hogar Agape Elim A.C la cual provee vivienda y alimentos a niños y adolescentes que sufrieron maltrato o abandono, y a madres solteras en situación de vulnerabilidad. Finalmente, la tercera entrevista se llevó a cabo en el Comedor San Vicente A.C. que provee alimentación a niños tarahumaras de escasos recursos cuya edad está entre 5 y 12 años.

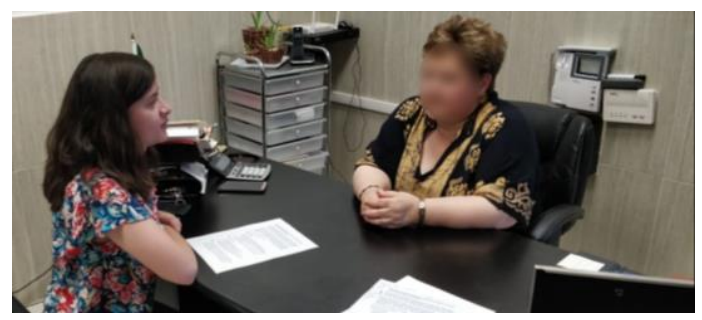

Figura 1. Entrevista semi-estructurada con responsable de la institución.

Posterior a las entrevistas, el equipo de trabajo se reunió para llevar a cabo un par de sesiones de interpretación contextual de entrevistas [9] y determinar las áreas de oportunidad, retos, y requerimientos. Con base a esta información se analizó y modeló el proceso de solicitud de donativos de alimentos y productos. A partir de este modelo y el entendimiento inicial, se llevaron a cabo sesiones de lluvia de ideas para el proceso de diseño. El presente trabajo únicamente plantea una de las ideas de las 3 que se discutieron en las sesiones de diseño

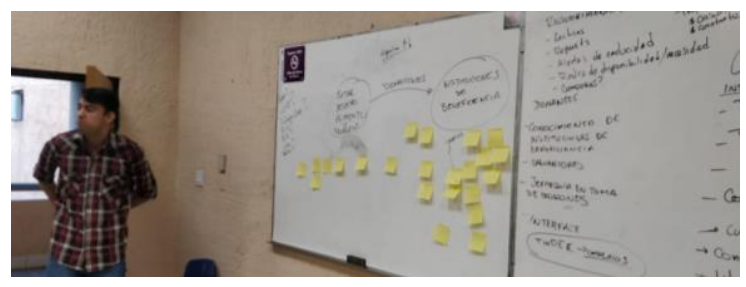

Figura 2. Sesión de diseño.

\section{Kuali}

Kuali es una aplicación móvil con la cual el usuario puede generar su propio perfil. Los perfiles se ajustan de acuerdo a si el usuario representa un corporativo (i.e. restaurantes, tiendas de conveniencia), particulares, o donatarios (i.e. casas hogares, comedores, o albergues). Con base a los requerimientos, Kuali busca facilitar y permitir a los usuarios ofrecer o solicitar productos de acuerdo a sus necesidades, así como facilitar la coordinación y distribución de las donaciones a las instituciones necesitadas.

El diseño de Kuali integra módulos de autenticación para asegurar que la ayuda llega en realidad a personas que lo requieren e identificar de manera apropiada a los donantes. Para ello, el diseño permite que el usuario ingrese la información requerida para la validación de su registro, tal como:

a. Nombres tanto de la institución como el representante legal de la misma.

b. Ubicación del inmueble.

c. Comprobante de su registro en el Padrón General para el Desarrollo Social.

Este proceso se realiza mediante el uso de recursos del dispositivo móvil, como la cámara para el reconocimiento de identificaciones oficiales (i.e. INE, Pasaporte, o permiso gubernamental para recibir donativos) y esta información se 
protege mediante técnicas de cifrados en servidores privados. De manera similar, Kuali utiliza el GPS del dispositivo móvil para ubicar físicamente el inmueble de la asociación donataria. Al finalizar el proceso de registro se muestran las pantallas personales de cada perfil de usuario. A continuación se describen cada una de ellas.
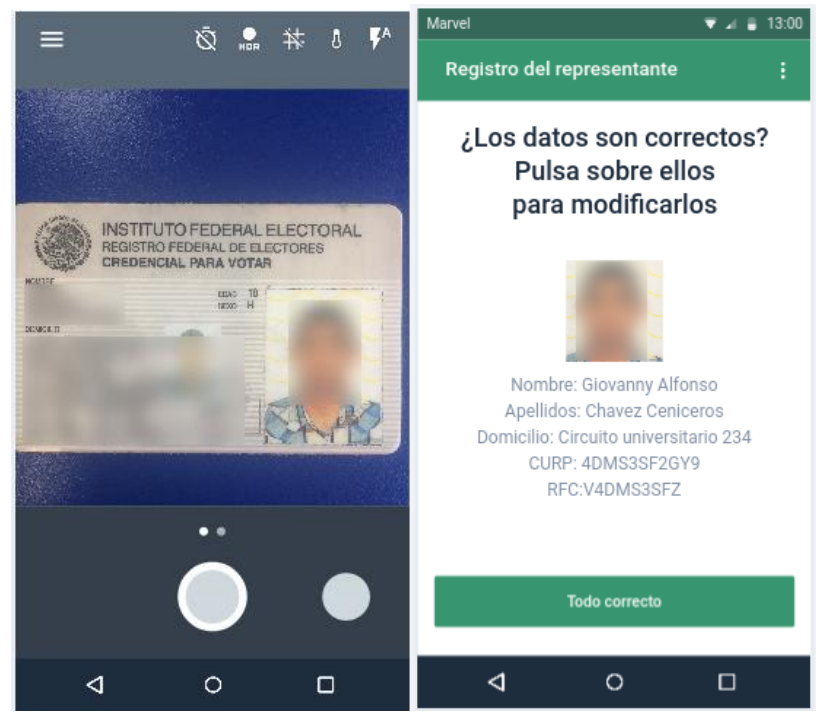

Figura 3. a) Captura de identificación, b) Confirmación de los datos

\subsection{Subsistema del donatario}

Consiguiente al proceso de autenticación, el donatario debe establecer sus necesidades recurrentes. Además, el usuario (representante de la institución donataria) puede solicitar alguna donación mediante el panel colocado a la izquierda de la aplicación. En este panel se encuentra un slider para ajustar el radio de búsqueda de donaciones (en kilómetros), un conjunto de representaciones gráficas del tipo de producto a solicitar y adicionalmente un selector de fecha límite para la petición (véase Figura 3a). Al completar los parámetros, el mapa (véase Figura 6). despliega los donantes localizados en el radio de búsqueda, cada uno con un perfil desplegable, con la posibilidad de entablar conexión para solicitar la donación (véase Figura 3b).
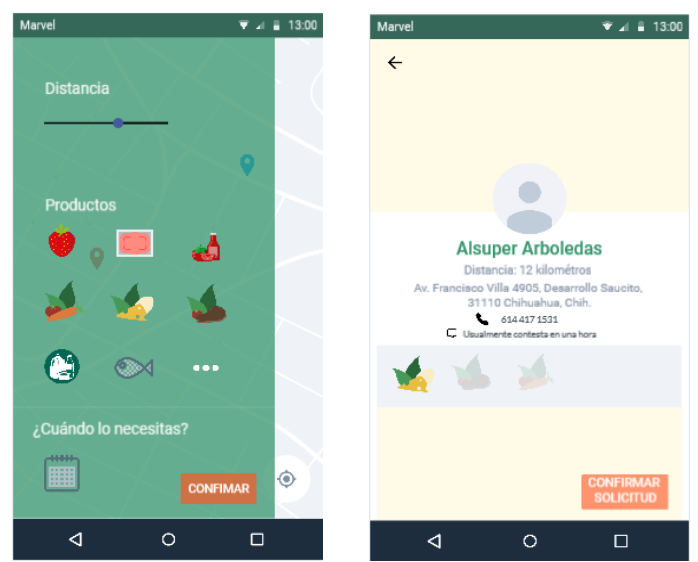

Figura 4. a) Selección del producto, b) Perfil del donante

Al realizar la solicitud el usuario debe esperar hasta que el donante acepte la petición. Al momento que el donante acepta, se establece un chat entre los interesados para ultimar detalles de la entrega y recepción del producto, así como posibles deducciones de impuestos (véase Figura 5a).

Finalmente, después de la entrega del producto solicitado, el donatario proporciona una calificación a la experiencia y servicio que tuvo con el donatario (véase Figura 5b).

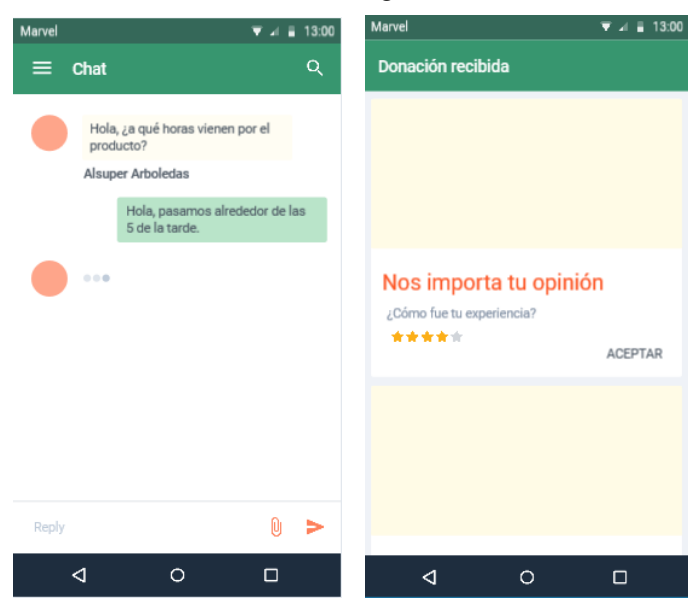

Figura 5. a) Chat de conexión entre donante y donatario. b) Calificación de partes para mantener una calidad el servicio de los usuarios de Kuali.

\subsection{Subsistema del donante}

Al momento de finalizar el registro, el donante puede observar un mapa de la ciudad (véase Figura 6). El donante puede especificar el producto que desea donar mediante el panel que se encuentra en lado izquierdo. Adicionalmente, en este panel se puede especificar la cantidad de producto que desea donar y la fecha de caducidad de dicho producto, la distancia (entre el donante y la instituciòn a donar) máxima a la cual puede trasladar el producto y por último si existe algún lugar donde prefiera donar (una casa hogar, un albergue, un comedor, etc.). Al confirmar lo anterior, el donante observa una vista actualizada del mapa en donde se muestran las ubicaciones de posibles lugares a los que puede donar de acuerdo a la información capturada por el donante.

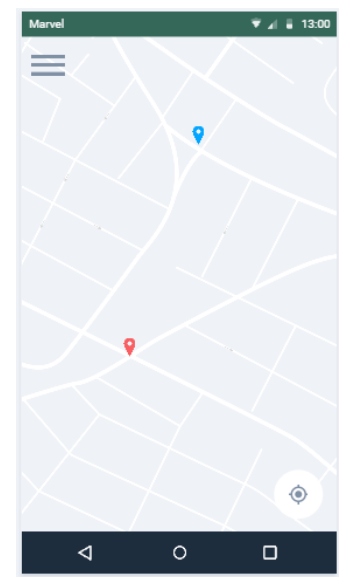

Figura 6. Mapa de la ciudad.

Una vez que el donante especifica la información anterior, Kuali permite al donante escoger alguna institución de su agrado o bien esperar que una institución solicite el apoyo que acaba de publicitar. Si el donante escoge una institución del mapa entonces Kuali muestra el perfil de la institución donde el donante puede revisar la información y confirmar si desea donar a ésta. Al 
confirmar, el donante debe esperar a que la institución que se seleccionó acepte el inicio de donación, el cual se refleja con un chat donde ambas partes se coordinan para establecer en el lugar y hora para la entrega de donaciones. Por el contrario, si el donante no selecciona una institución de preferencia, entonces Kuali publica la disponibilidad de la donación y el donante debe esperar a que soliciten su apoyo. Si el donante acepta hacer una conexión con alguna solicitud de un donatario entonces Kuali muestra el chat.

Al final, una vez que se haya hecho la entrega se le pide al donante calificar la experiencia que tuvo con la institución donde donó.

\section{Conclusiones y trabajo a futuro}

El desperdicio de alimentos es un problema de carácter mundial que tiene implicaciones sociales y económicas. Su solución puede llegar a ser muy compleja debido a diversos aspectos como la fecha previa de consumo, que sin duda debe ser seguida, la falta de información y comunicación entre instituciones donantes y donatarios.

El presente trabajo presenta una solución tecnológica que parte de dos problemas diferentes: el desperdicio alimentario y la falta de perecederos en albergues o instituciones afines. Por lo tanto, se realizaron entrevistas con representantes de algunas de estas instituciones para identificar requerimientos y obstáculos.

El diseño de Kuali presenta de manera general las necesidades de los donatarios y llegar al objetivo deseado: una herramienta capaz de aumentar los porcentajes de donaciones y satisfacer las necesidades alimentarias de las poblaciones vulnerables. Lo anterior se pretende lograr inicialmente mediante la agilización del proceso de donación y comunicación entre ambas partes.

Como trabajo futuro de este proyecto se pretende realizar el desarrollo de la aplicación móvil. Para esto será necesario llevar a cabo actividades centradas en el usuario para evaluar prototipos de baja y alta fidelidad. Durante la evaluación se contempla realizar entrevistas semi-estructuradas y sesiones think aloud para medir usabilidad, utilidad y eficiencia.. En concreto, re-diseñar y evaluar, no sólo la aplicación, también nuestras ideas para atacar este problema con la mayor eficacia posible.

\section{Agradecimientos}

Queremos agradecer a las instituciones que consistieron dar una entrevista y proporcionaron acceso a sus instalaciones para comprender con mayor detalle sus necesidades y retos. De igual manera queremos darle las gracias al alumno Oscar Eduardo Ordoñez Medina por su valiosa participación.

\section{Referencias}

[1] Parfitt, J., Barthel, M. \& Macnaughton, S. 2010. Food waste within food supply chains: quantification and potential for change to 2050, Phil. Trans. R. Soc., vol. 365, pp. 3065-3081

[2] FAO. 2011. Global food losses and food waste - Extent, causes and prevention. Rome

[3] FAO. 2014. Food losses and waste in Latin America and Caribbean. Rome

[4] FAO. 2016. Food losses and waste in Latin America and Caribbean. Rome

[5] Congreso del Estado de Chihuahua. 2018. Ley para la donación altruista de alimentos en el estado de Chihuahua, Chihuahua México, 30 de julio 2018

[6] Jahmeilah Roberson and Bonnie Nardi. 2010. Survival needs and social inclusion: technology use among the homeless. In Proc. of the conference on Computer supported cooperative work (CSCW '10). ACM, New York, NY, USA, 445-448. DOI: https://doi.org/10.1145/1718918.1718993

[7] Nisha Mohan and Abhraneel Sarma. 2017. Elevate: Ensuring Access to Food for Homeless Populations. In Proc of the CHI Conference Extended Abstracts on Human Factors in Computing Systems (CHI EA '17). ACM, New York, NY, USA, 80-85. DOI: https://doi.org/10.1145/3027063.3049274

[8] Rajasee S. Rege, Jennifer L. Allen, Eric P. Drewski, and Robert S. Molnar. 2008. The GroceryMate: eliciting community empathy and transforming it into purposeful action. In CHI '08 Extended Abstracts on Human Factors in Computing Systems (CHI EA '08). ACM, New York, NY, USA, 3885-3890. DOI: https://doi.org/10.1145/1358628.1358948

[9] Holtzblatt, K., Wendell, J. B., \& Wood, S. (2004). Rapid Contextual Design: A How-to Guide to Key Techniques for User-Centered Design. Elsevier Science. 